\title{
Performance analysis of NB-IOT network for patients monitoring in rural areas.
}

\author{
Athanase M.Atchome ${ }^{1}$,Frantz U. TOSSA ${ }^{1}$, Thierry O. Edoh ${ }^{2}$, Wahabou Abdou ${ }^{3}$, \\ Marc K. Assogba ${ }^{1}$, Eugene Ezin ${ }^{4}$,Antoine C. Vianou ${ }^{1}$. \\ 1 Ecole Doctorale des Sciences De l'Ingnieur(ED-SDI)/UAC/Benin, \\ 2 University of Bonn, Bonn, Germany, \\ 3 Université de Bourgogne, \\ 4 Institut de Formation et de Recherche en Informatique (IFRI/UAC)
}

\begin{abstract}
Medical surveillance is based on continuous monitoring of the patients vital parameters. The control of vital signs is essential in different cases. Actually, in the case of criticals healths issues (road or traffic accident; Pregnancy and pregnancy-related complication like blood pressure, foetal heart rate, etc...) vitals parameters need to be continually measured. Imagine the case where the patient is living in a rural area or remote area with poor health infrastructure. That kind of situation needs the involvement of news technologies (telemedicine, IoT), technics to help to overcome the medical service delivery issues. Base on this background and due to the technologies issues and limitations in some area or region the authors had proposed a new technology to support the medical service delivery at a remote area. The proposed technology consisted of Narrowband Internet of Things (NB-IoT). Its a promising technology that provides long-range communications at a low data rate for sensors with reduced device processing complexity and long battery lifetime. This paper aims to investigate the realistic performance of NBIoT in terms of effective throughput and patient served per cell in the healthcare monitoring system in a rural area with both in-band and stand-alone deployment.
\end{abstract}

Index Terms-Narrowband Internet of Things (NB-IoT); Healthcare monitoring; throughput; latency; device capacity; system-level analysis.

\section{INTRODUCTION}

The past quarter century has been a period of unprecedented change and progress in developing countries. Never the less this impressive progress, health access remains limited for many people especially, in rural area and slums in the large city. In fact, in Low and Middle-Income Countries (LMIC), the health sector is facing some challenges ( lack of skills, but also a shortage of health personnel and even worse, a lack of health centres). These issues are more several in the rural area. This compromises the provision, continuity and availability of care and services for the habitats of these areas. As a result, the populations in the areas face inadequate health care due to shortage or poor distribution of financial and human resources and a shortage of specialized services. Specialists often do not have enough "critical mass" of patients to be economically profitable to serve a region that is both sparsely populated and far away. The situation can be particularly difficult for patients with certain diseases, or for the elderly. Information and communication technology solutions, such as e-health, telemedicine, etc ... can be seen as ways to bridge the digital divide between rural and urban health centers and address deficiencies health sector in rural areas[6]. The term "rural" can be defined in a variety of ways, depending for example on the density of the population or geographical location. For example, the US Census Bureau defines rural as what is not urban - that is, after defining individual urban areas, what remains is rural [7]. In this article, we propose a network of NB-IOT sensors to coordinate the follow-up of the patients in a rural area of Benin and this from a health centre installed in this zone. The main contributions and results of this document are:

- Design a tractable wireless sensor network (NB-IOT) system to coordinate patient follow-up in a rural area of Benin;

- Remotely monitor the condition of critical patients;

- Enable the automatic collection of multi modal data, storage and processing using a single system over time;

- Increase the responsiveness of the nursing staff.

\section{NB-IOT OVERVIEW AND BACKGROUND}

In 3rd Generation Partnership Project (3GPP), a narrowband system based on Long Term Evolution (LTE) is being introduced to support the Internet of Things[1][2]. This system, named Narrowband Internet of Things (NB-IoT), can be deployed in three different operation modes [12]:

1) stand-alone as a dedicated carrier;

2) in-band within the occupied bandwidth of a wideband LTE carrier;

3) within the guard-band of an existing LTE carrier.

In the stand-alone operation mode, NB-IoT can occupy one GSM channel $(200 \mathrm{kHz})$ while for in-band and guard-band operation modes, it will use one physical resource block of LTE $(180 \mathrm{kHz})$. The design targets of NB-IoT include low-cost devices, high coverage (20-dB improvement over GPRS), long device battery life (more than 10 years), and massive capacity. Latency is relaxed although a delay budget of 10 seconds is the target for exception reports [8]. The NB-IOT design exploits the basic functionalities of LTE. However, the signalling and control channels for NB-IoT are new. Furthermore, in NBIoT, frequency division duplexing (FDD) half duplex type$\mathrm{B}$ is chosen as the duplex mode whereas legacy LTE also supports full duplex mode. FDD Half duplex means that updownlink are separated in frequency. Messages transfer in 
some subchannel is only unidirectional. User cannot perform both operations (transmission and reception) simultaneously [9]. The brief frame structure of both down-uplink along with the control channels, extracted from 3GPP standard, are as follows: The frame structure of NB-IoT downlink is similar to LTE in the time domain with $10 \mathrm{~ms}$ length. Each frame consists of 10 subframes of $1 \mathrm{~ms}$ length and each subframe consists of two slots with a length of seven OFDM symbols. In the frequency domain, it consists of one physical resource block (PRB) with 12 subcarriers having $15 \mathrm{kHz}$ of spacing and a normal cyclic prefix (CP)[11]. One sub-carrier one symbol constitute one resource element (RE), the smallest transmission unit. Furthermore, unlike LTE, NB-IoT has two physical signals and three physical channels which are as follows:

- Narrowband reference signal (NRS)

- Narrowband primary and secondary synchronization signals (NPSS and NSSS)

- Narrowband physical downlink control channel (NPD$\mathrm{CCH})$

- Narrowband physical downlink shared channel (NPDSCH)

In the uplink, NB-IoT support both single-and multi-tone transmissions. Multi-tone transmission uses the same SCFDMA scheme with a $15 \mathrm{kHz}$ subcarrier spacing. However, single-tone transmission support both $15 \mathrm{kHz}$ and $3.75 \mathrm{kHz}$ subcarrier spacing. The frequency $15 \mathrm{kHz}$ has similar numerology as in LTE. Whereas, the symbol duration of $3.5 \mathrm{kHz}$ subcarrier spacing has four times more duration compared to $15 \mathrm{kHz}$, which result in a slot length of $2 \mathrm{~ms}$. Furthermore, in NB-IoT uplink, a new resource mapping unit is defined as a resource unit (RU). RU is a combination of a number of subcarrier (frequency domain) and a number of slots (time domain). For the uplink, NB-IoT has one physical signal and two physical channels which are as follows:

- Demodulation reference signal (DMRS)

- Narrowband physical random access channel (NPRACH)

- Narrowband Uplink Shared Channel (NPUSCH)

NB-IoT is a Low Power WAN radio standard (LPWAN) developed by $3 \mathrm{GPP}$ to enable a wide range of cellular devices and services [10]. Key target applications include smart cities, personal IoT applications, smart grids, smart meters, logistics, industrial monitoring, agriculture, and more. In this work, we used the NB-IoT application in the Wireless Remote Monitoring System (WBAN). These systems are wireless sensors that communicate over a long-range wireless link to send or receive information.

\section{APPLICATION USE-CASE: HEALTH CARE MONITORING SYSTEM}

In hospitals, the temperature of a patients body, for instance, need to be monitored constantly, which is generally made by the staff members of the hospital. They notice the temperature of the patients body constantly and keeps a record of it. Health monitoring means monitoring a person to identify any changes in his or her health status because of exposure to certain health hazards arising from the conduct of the business or undertaking (GRWM Regulations). Health monitoring is a way to check if the health of workers is being harmed from exposure to hazards while carrying out work, and aims to detect early signs of ill-health or disease. Health monitoring can show if control measures are working effectively. Monitoring does not replace the need for control measures to minimise or prevent exposure. Nowadays, health care sensors are playing an essential role in hospitals. The patient monitoring system is one of the major developments because of its innovative technology. An automatic wireless health monitoring system is used to measure the patients body temperature and heartbeat by using embedded technology.

The use of NB-IoT allows the use of an already deployed cellular base station and covers all facilities in underdeveloped countries, eg rural hospitals. NB-IoT offers end-user terminals (eg sensor nodes) a long service life. However, each application is characterized by different coverage requirements and performance requirements in terms of tattoo demand or latency. For example, health usually requires monitoring of perspiration, respiratory rate, body temperature, pulse and blood pressure, etc. Data rates of up to 2 Kbps per sensor may be required. In our design model of the health care monitoring system, we consider the single-sensor nodes. In this design, each sensor node, such as a temperature sensor, a respiratory rate sensor, etc., is considered an individual node and each node has its own transmission module. Therefore, each node transmits data to the central processing unit through the eNB with latency and data rate requirements [4]. In this design, for each patient, several transmission links are needed with the base station. The traffic in the health care monitoring system is based on multiple sensors with different sizes of information packets and time interval. In our analysis, we assume the case of a critical patient requiring constant surveillance. All sensors communicate with the NB-IoT base station directly for each patient.In this context, all patient data is sent by the sensors carried by the patients to the treatment center via the base station. These data are processed and health staff such as general practitioners, specialists, nurses, carer can consult, use in real time. Fig 1. shows the proposed model.

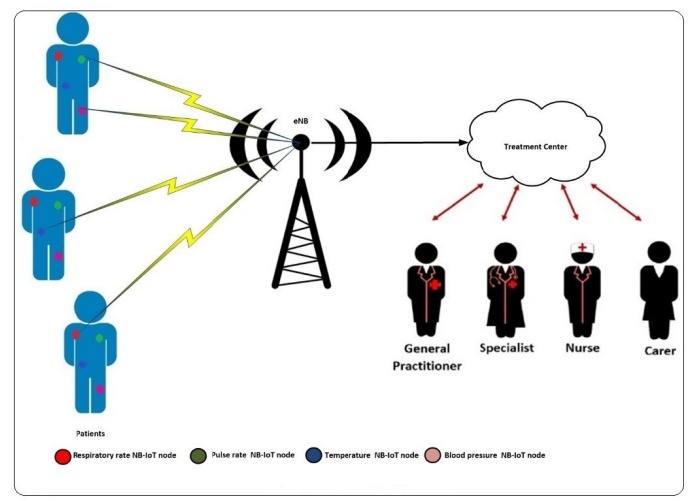

Fig. 1. Overview of the proposed model 


\section{METHODOLOGY, DATA AND SYSTEM MODEL}

To conduct the performance analysis, we have considered both in-band and standalone deployments of NB-IoT with a bandwidth of $180 \mathrm{kHz}$ in a typical LTE. The scenario is a regular grid of tri-sector sites with inter-site distance of $1732 \mathrm{~m}$. In-band mode, for instances where cellular services are present and NB-IoT is positioned in the LTE carrier sharing LTE resources; this mode of operation is perhaps the more costeffective and seamless for mobile operators since it does not require any hardware changes of the radio access network, and efficiently uses spectrum resources for LTE or NB-IoT services based on demand from mobile users or devices. In Standalone mode, for instances where cellular services are not present or are decommissioned to make narrowband spectrum available, which is the case of cellular GSM; by reframing one or more GSM carriers to carry NB-IoT traffic, operators can ensure a smooth transition to LTE for massive machine type communication. The Fig 2 illustrate the deployment of in-band and Stand Alone.

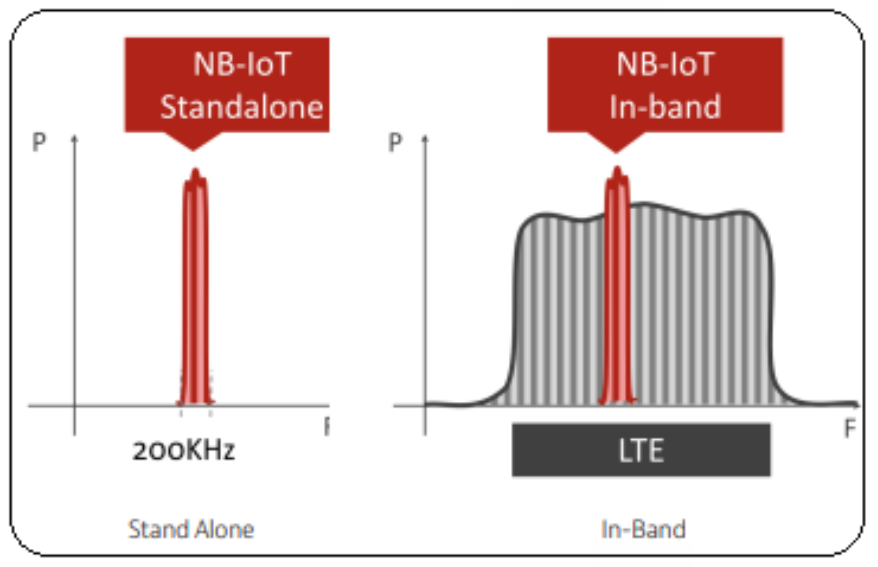

Fig. 2. in-band and Stande Alone

The other simulation assumptions that closely follows are presented in Table1. The full $180 \mathrm{kHz}$ bandwidth, i.e. 12 subcarriers at $15 \mathrm{kHz}$ subcarrier spacing in both downlink and uplink, is used for the analysis. For uplink, this is known to perform worse than single tone e.g. $3.75 \mathrm{kHz}$ or $15 \mathrm{kHz}$, so the achieved performance in this study yields lower bounds as compared to what can be achieved with single tone NB-IoT systems and it is more realistic assumption to investigate NB-IoT uplink performance[3]. The data rate $R_{i, n}$ for a node $\mathrm{i}$ when the $n_{t h}$ PRB is assigned is given by:

$$
R_{i, n}=\operatorname{Blog}_{2}\left(1+S I N R_{i, n}\right)
$$

where $\mathrm{B}$ is the assigned transmission bandwidth and $S I N R_{i, n}$ is the signal-to-interference-plus-noise (SINR) for node $\mathrm{i}$ on $n_{\text {th }}$ PRB and is given as:

$$
S I N R_{i, n}=\frac{P_{n} h_{i, n}}{I_{i, n}+N_{0}}
$$

where $P_{n}$ is the transmit power and $h_{i, n}$ is the channel gain between node $\mathrm{i}$ and the base station on the nth PRB. $I_{i, n}$ is the interference experienced by node $\mathrm{i}$ and is assumed to be negligible in our analysis. $N_{0}$ is the noise power spectral density. Based on the SINR, the corresponding MCL is computed. The relationship between SINR and MCL is given as [4]:

$$
\text { TargetSINR }=T_{\text {xpower }}+174-\text { Noisefigure }
$$

$-10 \log _{10}(B)-M C L$

The path loss is given by:

$$
\text { PathLoss }=I+37.6 \log _{10}(R)
$$

$\mathrm{R}$ in Kilometers with $\mathrm{I}=120.9$ for the $900 \mathrm{Mhz}$

The inter-arrival time is distributed over three categories of periodic transmissions with constant inter-arrival times of 1 day, 2 hours, 1 hour and 30 minutes. Based on [4],the respective proportions of devices are $46 \%, 47 \%, 15 \%$, and $5 \%$. The average arrival rate of reports per device is:

$$
\frac{0.46}{86400 s}+\frac{0.47}{7200 s}+\frac{0.15}{3600 s}+\frac{0.005}{1800 s}=129.6 * 10^{-6 \frac{\text { packet }}{s} / \text { cell }}
$$

The total rate of uplink packets in the network is:

21 cell $/$ network $* 6.82 \frac{\text { packet }}{\mathrm{s}} /$ cell $=143.0 \frac{\text { packet }}{\mathrm{s}} /$ network

The reverse calculation from network packet rate, $\mathrm{R}$ [reports/s/cell], to number of devices is:

$$
\frac{R^{\frac{\text { packet }}{s}} / \text { cell }}{129.6 * 10^{-6 \frac{\text { packet }}{s}} / \text { device }}=7716 * \text { R.device } / \text { cell }
$$

For the simulation, chase combining is used based on MCL, such that the same information is repeated $\mathrm{N}$ times, $\mathrm{N}$ being the number of repetitions. The number of repetitions assumed with different MCL values are presented in Table1.

\begin{tabular}{|l|c|}
\hline Parameters & assumptions \\
\hline \hline simulation frequency & $24 \mathrm{GHz}$ \\
\hline channel Bandwidth & $900 \mathrm{MHz}$ \\
\hline inter-site distance & $1732 \mathrm{~m}$ \\
\hline eNB transmit power & $32 \mathrm{dBm}$ \\
\hline user transmit power & $23 \mathrm{dBm}$ \\
\hline shadowing standard standard deviation & $8 \mathrm{~dB}$ \\
\hline shadowing correlation distance & $110 \mathrm{~m}$ \\
\hline shadowing correlation between cell-sites & 0.5 \\
\hline shadowing correlation between cell sector & 1.0 \\
\hline building penetration loss & $40 \mathrm{~dB}$ \\
\hline noise figure at user equipment & $3 \mathrm{~dB}$ \\
\hline noise power spectral density & $-174 \mathrm{dBm} / \mathrm{Hz}$ \\
\hline
\end{tabular}




\section{RESULTS AND DISCUSSION}

In this section, the performance of the NB-IoT system-level system is evaluated using Monte Carlo simulations in terms of actual throughput and the number of devices supported. With the parameters shown in Table 1, the simulation is run for band and standalone deployments for more than 750 random samples. Figure 3 illustrates the cumulative distribution function (CDF) of the average effective flow in different strip and stand-alone deployments. The actual rate is defined as the number of bits of information transmitted per second with all the control information headers. The tape deployment performance in terms of battery life is significantly degraded due to the presence of LTE control information and the different interferences due to the two coexisting systems. Figure 4 shows the average number of patients that can be treated in different deployment scenarios. It can be seen that autonomous deployment is an important way to dramatically improve the flow and number of patients in care.

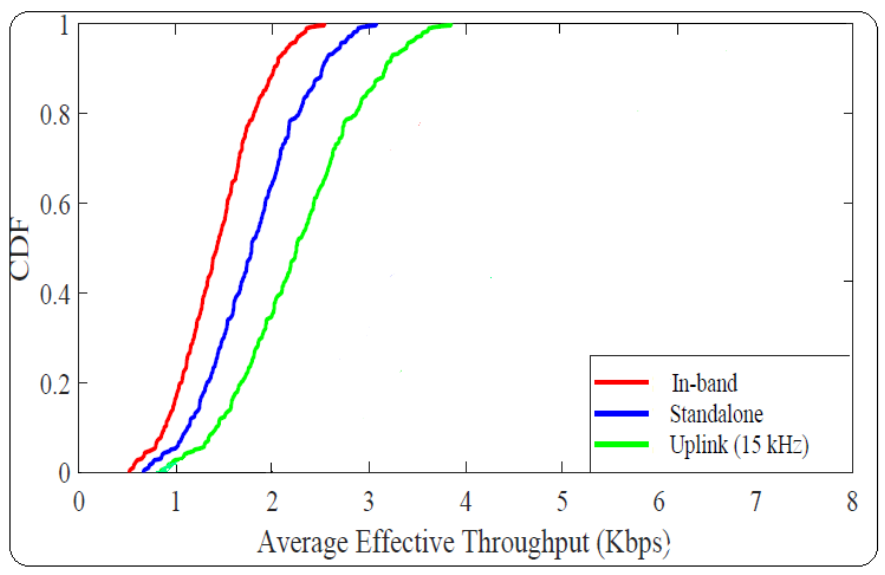

Fig. 3. Throughput in standalone and in-band

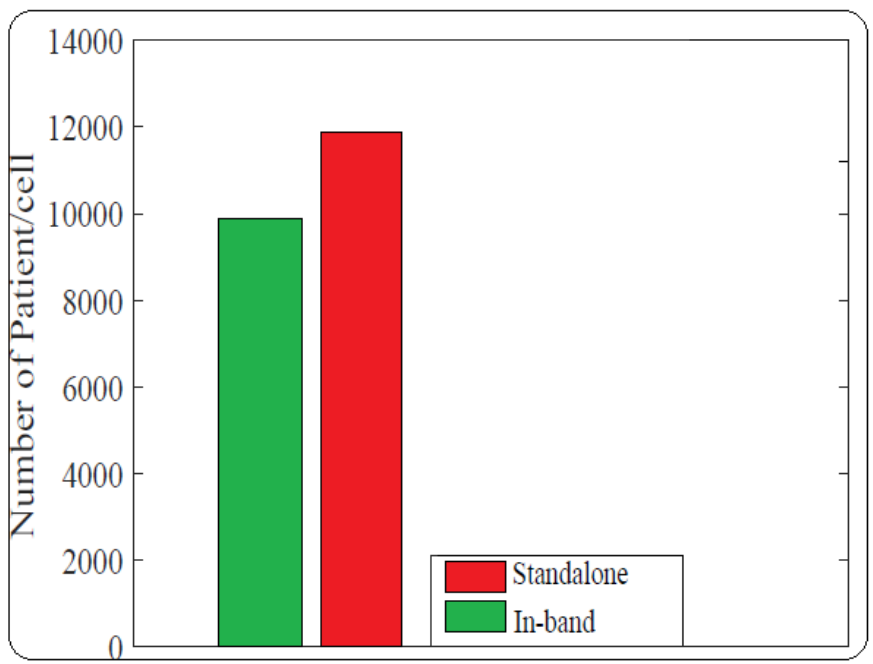

Fig. 4. Device per cell

\section{CONCLUSION AND PERSPECTIVES}

In this article, we presented a detailed performance analysis specific to a NB-IoT application for a rural health care surveillance system. The analysis carried out shows that with the autonomous deployment, one observes a significant gain in flow and a large number of patients per cell compared to the deployment in band. From these results, we can conclude that the NB-IoT technology is better suited for monitoring patients in rural areas, due to precarious conditions such as lack of electricity and medical infrastructure. Based on the results of this work, we can in terms of perspectives, work to precisely determine the geolocation of patients and evaluate different resource management strategies in NB-IoT rural systems.

\section{REFERENCES}

[1] 3GPP TR 45.820. Cellular System Support for Ultra Low Complexity and Low Throughput Internet of Things. Technical Report; 2015.

[2] 3GPP R1157398. NB-IoT - System level evaluation and comparison standalone. Technical Report; Ericsson; 2015.

[3] 3GPP R1157248. NB-IoT Capacity evaluation. Technical Report; Nokia Networks; 2015.

[4] Persia S, Rea L. Next generation M2M Cellular Networks: LTEMTC and NB-IoT capacity analysis for Smart Grids applications. AEIT International Annual Conference (AEIT) 2016; p. 16.

[5] Berliandhy IE, Rizal A, Hadiyoso S, Febyarto R. A multiuser vital sign monitoring system using ZigBee wireless sensor network.International Conference on Control, Electronics, Renewable Energy and Communications (ICCEREC) 2016; p. 136140.

[6] Nkqubela L Ruxwana, Marlien E Herselman and D Pieter Conradie, 'ICT applications as e-health solutions in rural healthcare in the Eastern Cape Province of South Africa", HEALTH INFORMATION MANAGEMENT JOURNAL Vol 39 No 12010 ISSN 1833-3583 (PRINT) ISSN 1833-3575 (ONLINE)

[7] Michael Ratcliffe, Charlynn Burd, Kelly Holder, and Alison Fields, "Defining Rural at the U.S. Census Bureau", in American Community Survey and Geography Brief, Issued December 2016

[8] Rapeepat Ratasuk, Benny Vejlgaard, Nitin Mangalvedhe and Amitava Ghosh, "NB-IoT system for M2M communication", in IEEE Wireless Communications and Networking Conference, 2016

[9] Hassan Malik, Muhammad Mahtab Alam, Yannick Le Moullec and Alar Kuusik, "NarrowBand-IoT Performance Analysis for Healthcare Applications" 9th International Conference on Ambient Systems, Networks and Technologies, ANT-2018 and the 8th International Conference on Sustainable Energy Information Technology, SEIT 2018, 8-11 May, 2018, Porto, Portugal

[10] Usman Raza, Parag Kulkarni, and Mahesh Sooriyabandara "Low Power Wide Area Networks: An Overview", IEEE Communications Surveys \& Tutorials, 2017

[11] Y.-P. Eric Wang, Xingqin Lin, Ansuman Adhikary, Asbjrn Grvlen, Yutao Sui,Yufei Blankenship, Johan Bergman and Hazhir S. Razaghi, "A Primer on 3GPP Narrowband Internet of Things (NB-IoT)" IEEE Communications Magazine (Volume: 55 , Issue: 3 , March 2017 )

[12] Alexandros-Apostolos A. Boulogeorgos, Panagiotis D. Diamantoulakis, George K. Karagiannidis, "Low Power Wide Area Networks (LPWANs) for Internet of Things (IoT) Applications: Research Challenges and Future Trends" Published 2016 in ArXiv 